\title{
Sizing up social interaction
}

DOI:

10.1038/nrn1941

URLs
Individuals with mutations in the tumour suppressor gene PTEN are prone not only to tumours but also to brain disorders, including macrocephaly (enlarged head circumference), seizures and mental retardation. Although PTEN mutations have been reported in autistic patients with macrocephaly, it is not clear whether there is a causal link between this gene and autistic spectrum disorders (ASD). Writing in Neuron, Chang-Hyuk Kwon and colleagues provide direct evidence that inactivation of Pten in mice results in neuropathological changes as well as abnormalities in social interaction.

Mice lacking Pten die during embryogenesis, so the researchers used a conditional knockout approach to study the role of

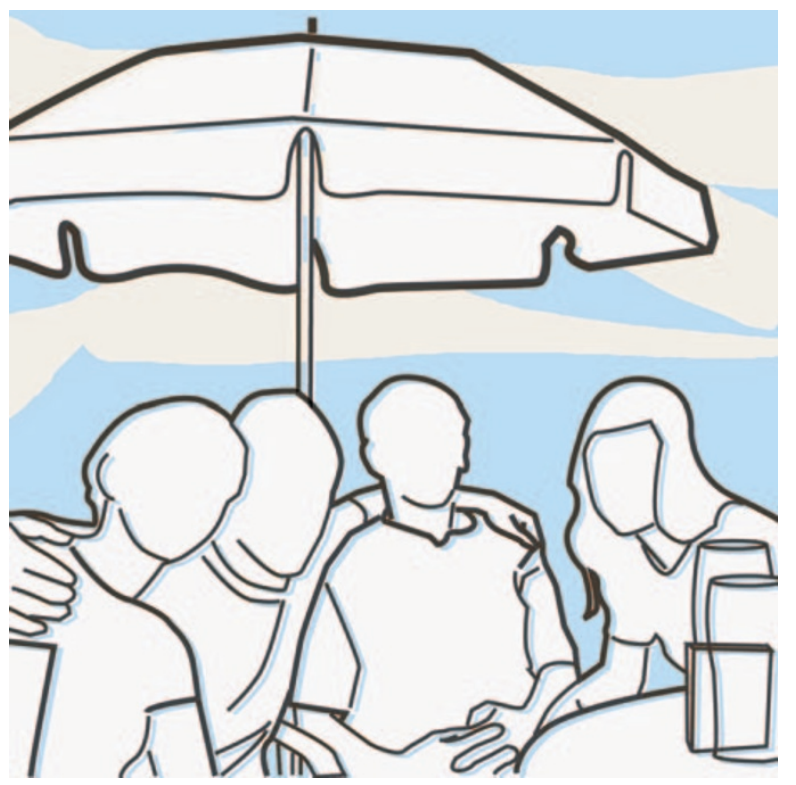

PTEN in mature neurons in which specialized properties, such as synapses and polarity, are well established. They eliminated Pten in discrete mature neuronal populations in the cerebral cortex and hippocampus. The mutant mice showed progressive soma hypertrophy and macrocephaly that were largely confined to the forebrain, in which most Pten deletions occurred.

As PTEN signalling has been implicated in neurite outgrowth, Kwon et al. investigated the effects of Pten loss on existing neuronal processes. In the mutant animals, there were ectopic granule axons, a dramatic enlargement of the mossy fibre tract, and a marked increase in the number of presynaptic vesicles. Golgi staining revealed dendritic hypertrophy characterized by an increase in dendritic thickness and spine density. Furthermore, there was an elevation in the downstream signalling components of the PI3K (phosphatidylinositol-3-kinase) pathway, which is consistent with the involvement of PI3K in synaptic plasticity and the fact that PTEN inhibits PI3K signalling.

Using several behavioural models, the researchers show that the Pten-mutant mice have deficits in social learning and interaction. For example, the mutant animals spent less time investigating the social target (a new mouse) compared with controls. When presented with a choice between the social target and an inaminate object, the Pten mutants spent similar amounts of time interacting with both. When the social target was removed and later re-introduced, the mutant mice, unlike their normal counterparts, did not reduce their interaction with it, indicating that they might have impaired social learning.

Kwon and co-workers next asked whether the abnormalities in social interaction in Pten-mutant mice were due to sensory and/or locomotor dysfunctions. The animals showed normal responses in several behavioural assays such as locomotor activity, motor coordination and fear conditioning. However, they had hyperactivity, increased startle responses, and deficits in transmission of sensory information to the motor system, as well as increased anxiety-like responses under some conditions.

As the Pten conditional mutants show phenotypes similar to autistic patients with macrocephaly, the researchers suggest that they have produced a potential animal model for this disorder. Whether this is the case remains to be seen. Nevertheless, the findings are intriguing, and might be important for identifying molecular mechanisms underpinning social- and anxiety-related behaviours in the adult central nervous system.

Jane Qiu

ORIGINAL RESEARCH PAPER Kwon, C.-H. et al. Pten regulates neuronal arborization and social interaction in mice. Neuron 50, 377-388 (2006) WEB SITE

Parada's laboratory: http://www8. utsouthwestern.edu/utsw/cda/dept24916/ files/72320.html 